\title{
Biphasic Proton Transport Mechanism for Uncoupling Proteins
}

Afshan Ardalan ${ }^{1}$, Shahin Sowlati-Hashjin ${ }^{2,3}$, Habib Oduwoye ${ }^{1}$, Stephanie O. Uwumarenogie ${ }^{1}$, Mikko Karttunen ${ }^{2,3,5}$, Matthew D. Smith ${ }^{4}$, and Masoud Jelokhani-Niaraki ${ }^{1 *}$

${ }^{1}$ Department of Chemistry and Biochemistry, Wilfrid Laurier University, Waterloo, ON, Canada N2L 3C $5 ;{ }^{2}$ Department of Chemistry, The University of Western Ontario, London, ON, Canada N6A 3K7; ${ }^{3}$ The Center for Advanced Materials and Biomaterials Research, The University of Western Ontario, London, ON, Canada N6K 3K7; ${ }^{4}$ Department of Biology, Wilfrid Laurier University, Waterloo, ON, Canada N2L 3C5; ${ }^{5}$ Department of Physics and Astronomy, The University of Western Ontario, London, ON, Canada N6A 3K7

\section{Table of Contents}

Table S1 Proton transport rate of UCP2 and its mutants

Table S2 Water Permeability of UCP2 subunits $\quad$ S2

Figure S1 Alternating access mechanism $\quad$ S3

Figure S2 Western Blot and Coomassie blue stained semi-native SDS-PAGE $\quad$ S3

Figure S3 Schematic representation of the prepared proteoliposome $\quad$ S4

Figure S4 Final structure of UCP2, DN, 2DN, 3DN, 3KQ, and Hexamutant subunits S5

Figure S5 RMSD of the backbone atoms for the tetrameric UCP2 systems

Figure S6 Solvent accessible surface area for all studied systems $\quad$ S6 
Table S1- Proton transport rate of UCP2 and its mutants in the absence and presence of 500 $\mu \mathrm{M}$ ATP. All the units are in $\mu \mathrm{mol}$ of proton $/ \mathrm{min} / \mathrm{mg}$ protein

\begin{tabular}{ccc}
\hline protein & $\begin{array}{c}\text { proton } \\
\text { transport rate } \\
\text { (no ATP) }\end{array}$ & $\begin{array}{c}\text { proton } \\
\text { transport rate } \\
\mathbf{( 5 0 0} \boldsymbol{\mu M} \text { ATP) }\end{array}$ \\
\hline UCP2 & $5.24 \pm 0.09$ & $1.17 \pm 0.09$ \\
D35N & $10.43 \pm 0.16$ & $3.91 \pm 0.01$ \\
K141Q & $8.53 \pm 0.12$ & $3.25 \pm 0.32$ \\
D138N & $13.83 \pm 1.06$ & $5.64 \pm 0.23$ \\
K239Q & $11.38 \pm 0.81$ & $4.34 \pm 0.20$ \\
D236N & $10.62 \pm 0.94$ & $4.90 \pm 0.28$ \\
K38Q & $13.33 \pm 0.70$ & $6.03 \pm 0.54$ \\
2DN & $16.14 \pm 0.93$ & $7.67 \pm 0.77$ \\
2KQ & $12.41 \pm 1.29$ & $4.94 \pm 0.35$ \\
3DN & $31.26 \pm 0.95$ & $15.97 \pm 0.55$ \\
3KQ & $11.82 \pm 0.48$ & $5.21 \pm 0.95$ \\
Hexamutant & $6.21 \pm 0.06$ & $1.89 \pm 0.11$ \\
\hline
\end{tabular}

Table S2- Water Permeability $\left(\mathrm{cm}^{3} / \mathrm{s}\right)^{*}$ of UCP2 subunits during the last $10 \mathrm{~ns}$ of simulation (240 $-250 \mathrm{~ns})$

\begin{tabular}{ccccc}
\hline & Subunit 1 & Subunit 2 & Subunit 3 & Subunit 4 \\
\hline $\mathbf{R}_{\mathbf{t}}\left(\mathbf{s}^{-1}\right)$ & $2 \times 10^{8}$ & $2 \times 10^{8}$ & $3 \times 10^{8}$ & $1 \times 10^{8}$ \\
$\mathbf{P}_{\mathbf{f}}\left(\mathbf{c m}^{\mathbf{3}} / \mathbf{s}\right)$ & $5.98 \times 10^{-15}$ & $5.98 \times 10^{-15}$ & $8.97 \times 10^{-15}$ & $2.99 \times 10^{-15}$ \\
\hline
\end{tabular}

*Permeability was calculated according to $P_{f}=v_{w} R_{t} / N_{A}$ where $v_{w}$ is the average volume of a water molecule $\left(18 \mathrm{~cm}^{3} / \mathrm{mol}\right), \mathrm{R}_{\mathrm{t}}$ is the water transport rate, and $\mathrm{N}_{\mathrm{A}}$ is the Avogadro's number. The plane of three residues I43, A146, and N244 on the matrix-side of UCP2 was selected and water transfer across the plane was monitored. 


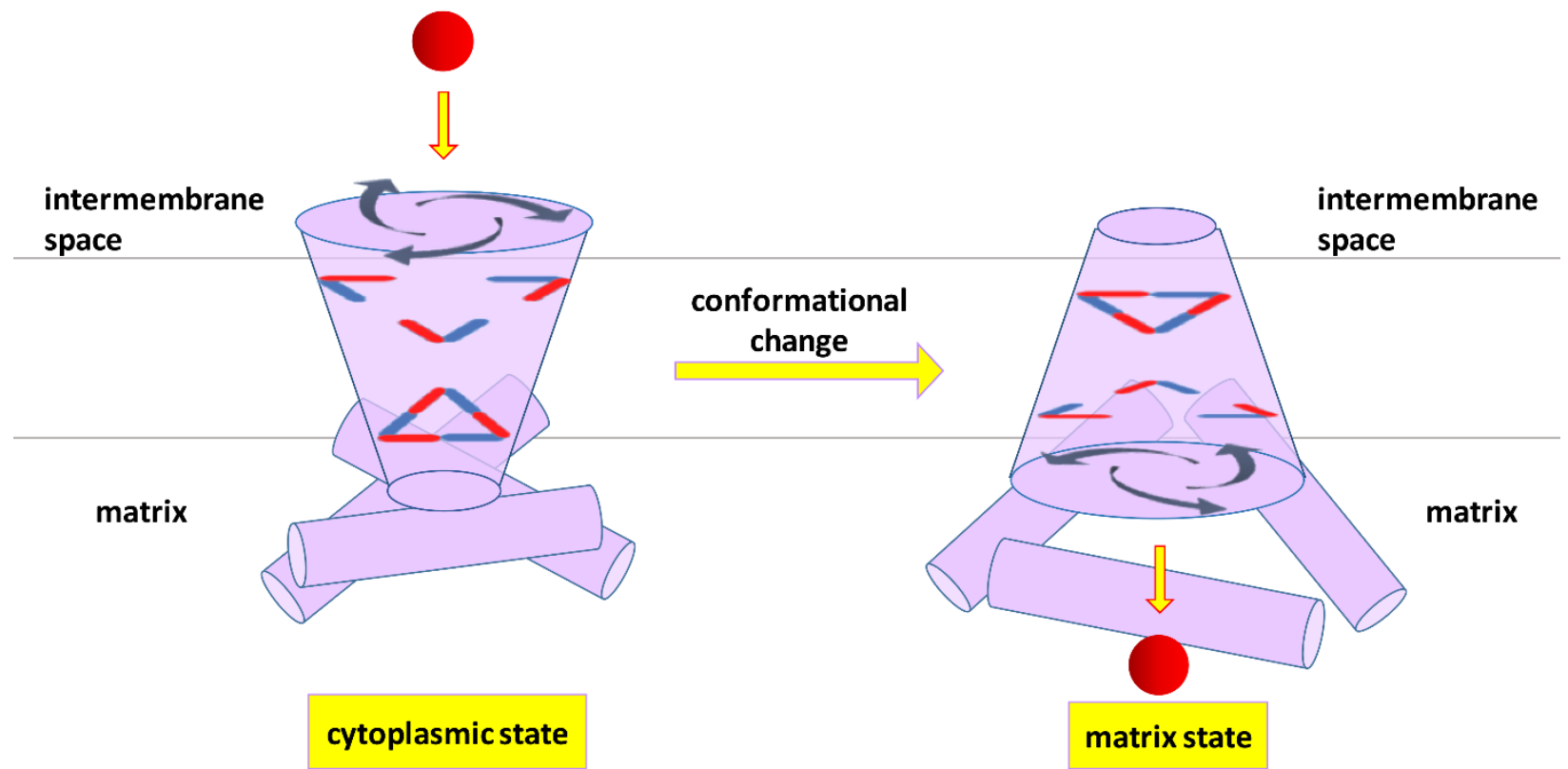

Figure S1- Alternating access mechanism is proposed to be a common mechanism for substrate translocation by members of MCF: cavity opening alternates between being towards the cytoplasmic and matrix side.

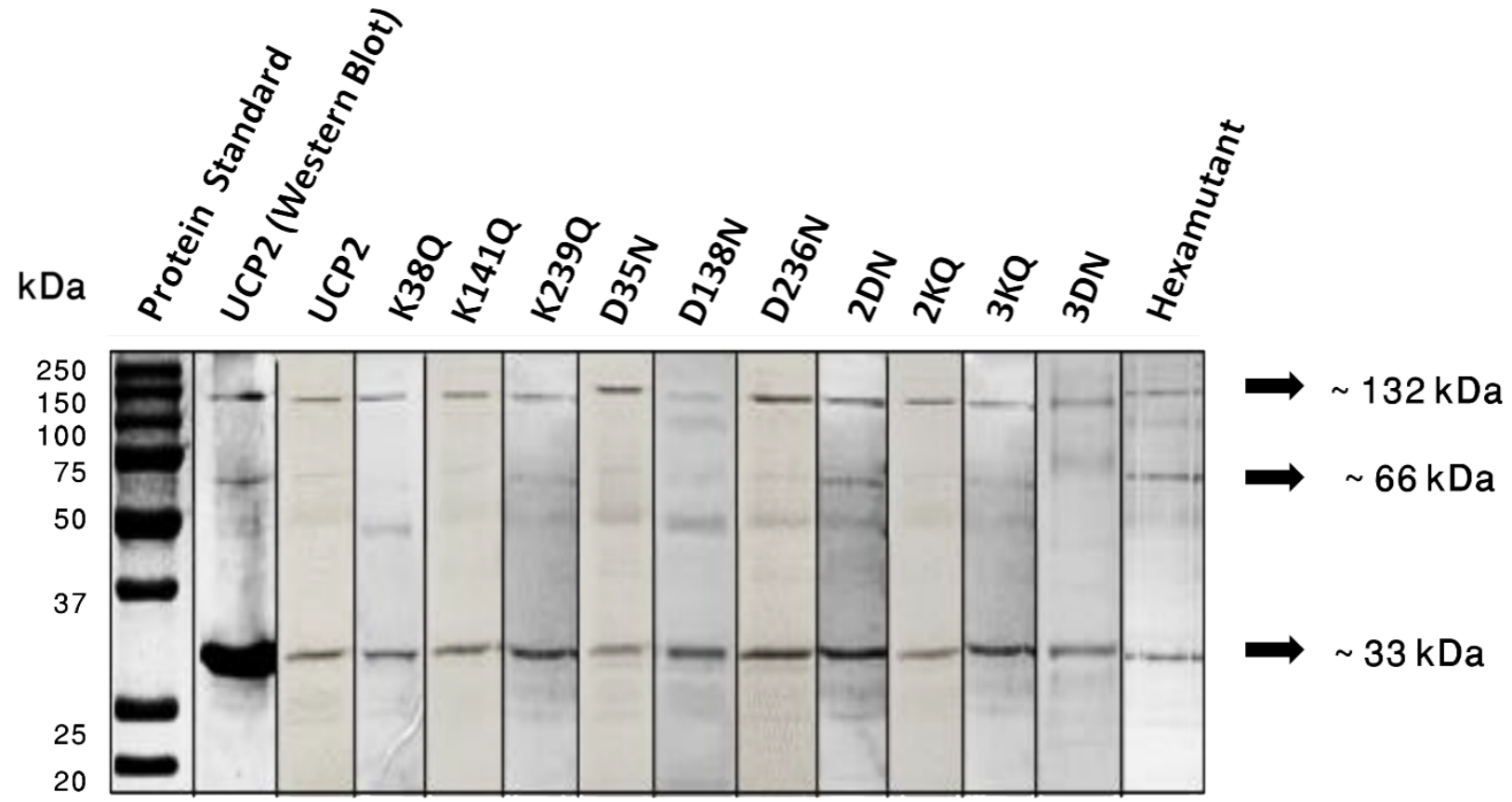

Figure S2- Western Blot and Coomassie blue stained semi-native SDS-PAGE analysis of purified monomeric and oligomeric UCP2 and its mutants in 1\% OG 

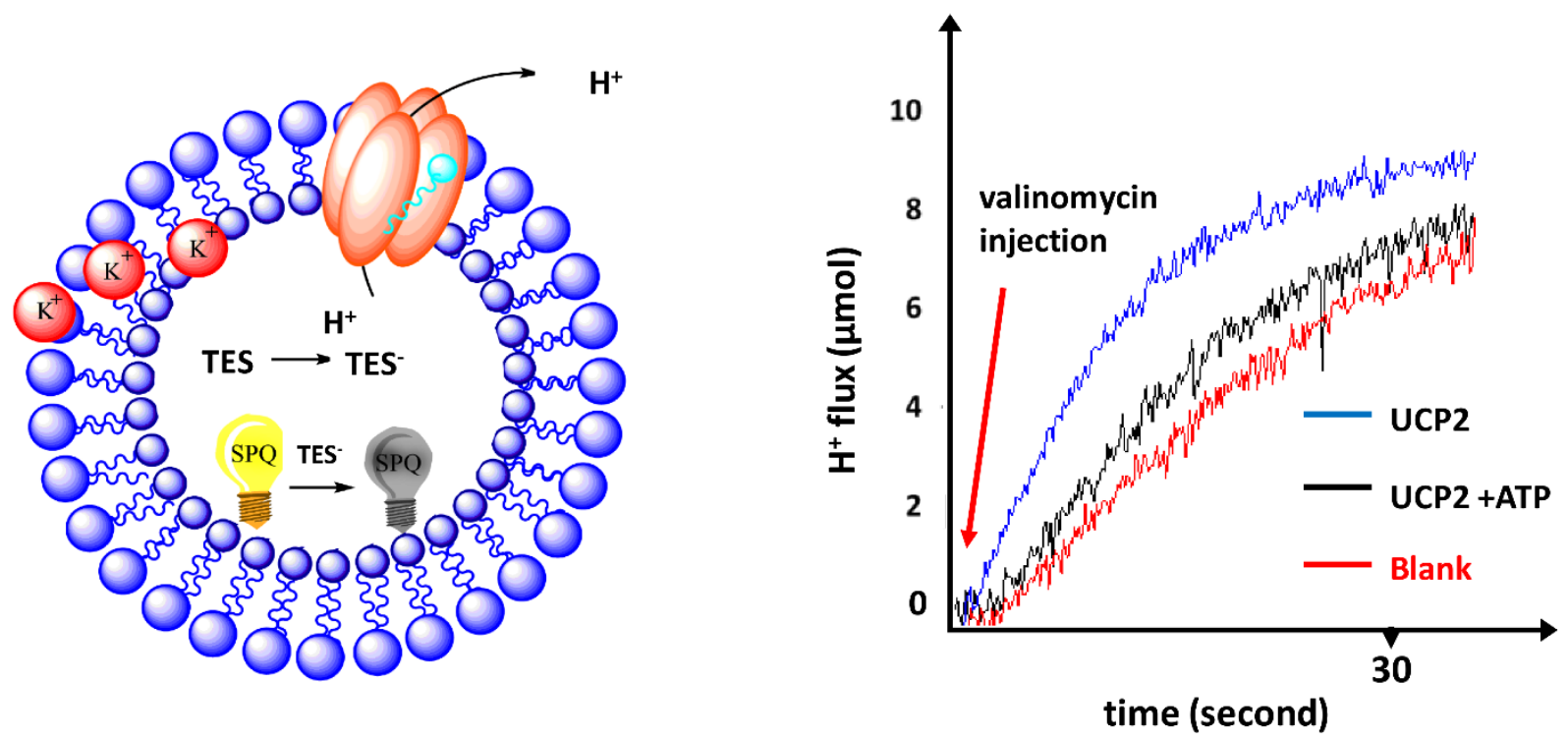

Figure S3- (a) Schematic representation of the prepared proteoliposome for measuring the rate of proton transport by fluorescence quenching assay. The trapped SPQ fluorescent dye inside the vesicles can be quenched by $\mathrm{TES}^{-}$anions. TES ${ }^{-}$anions are generated as a consequence of increase in internal $\mathrm{pH}$. Valinomycin directs the potassium ions into the liposomes resulting in an uneven charge distribution (inside positive) and osmotic imbalance across the lipid bilayer, which causes a fatty acid-dependent UCP2-mediated proton efflux. Thus, the rate of proton transport is indirectly measured by the rate of quenching of the SPQs fluorescence signal. (b) UCP mediated proton transport in the absence and presence of ATP and non-specific proton flux are shown for comparison. 
(I)
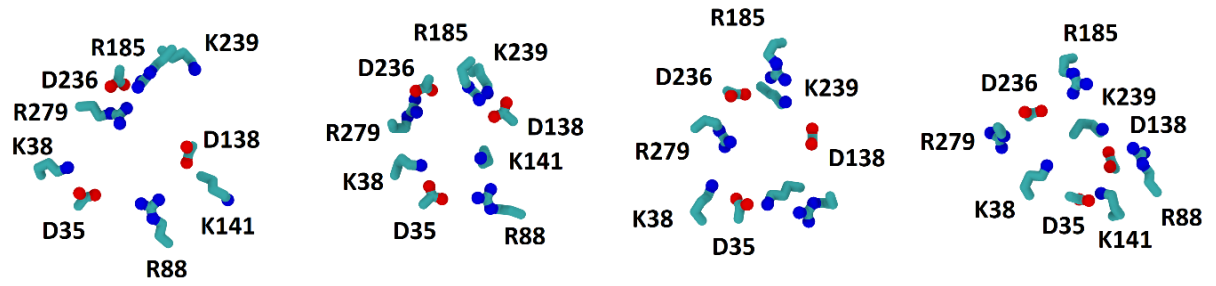

(II)
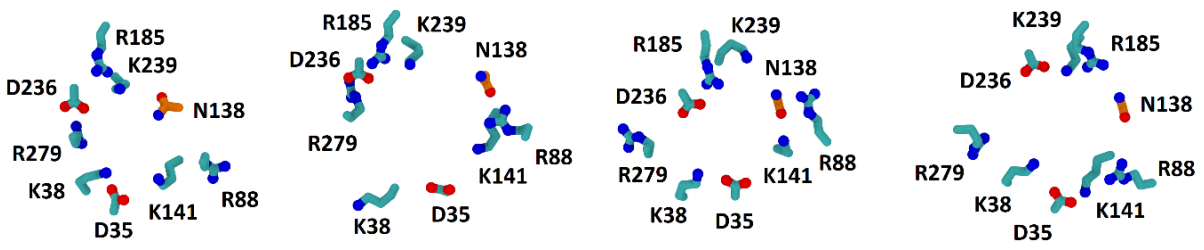

(III)
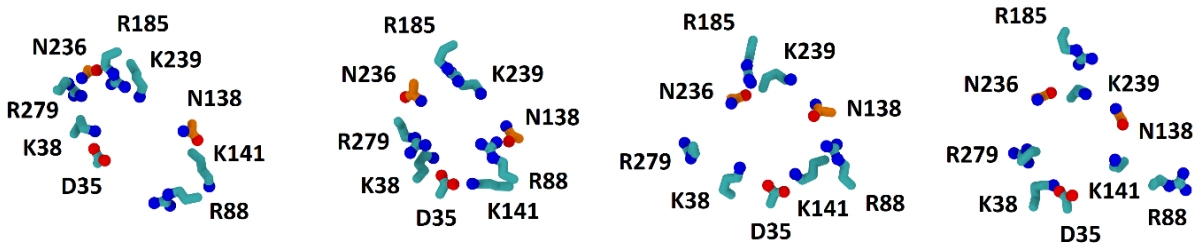

(IV)
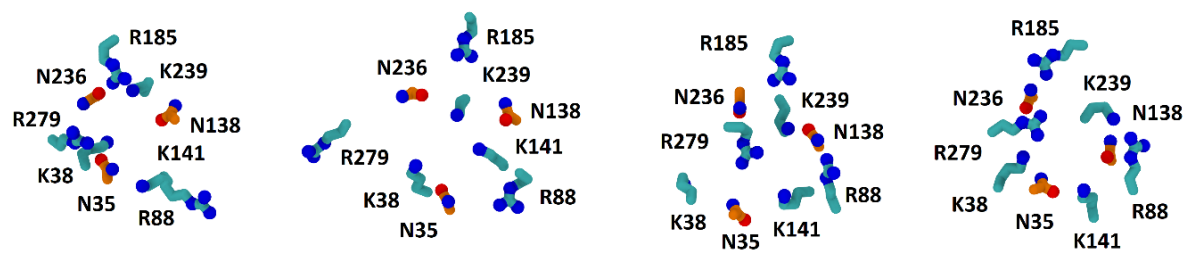

(V)
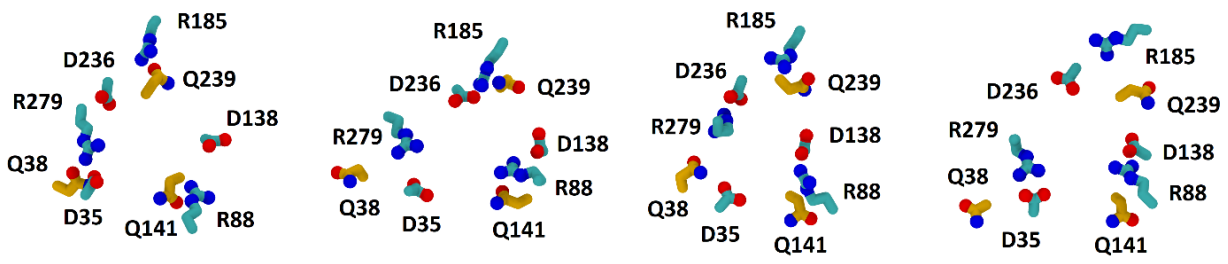

(VI)
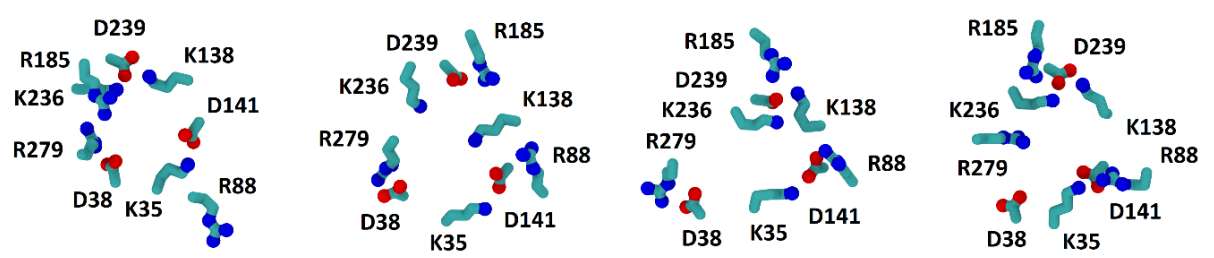

Figure S4- Top views of the final structures of (I) UCP2, (II) DN, (III) 2DN, (IV) 3DN, (V) 3KQ, and (VI) hexamutant subunits (A, B, C and D from left to right) at the site of the matrix network. $D \rightarrow N$ and $\mathrm{K} \rightarrow \mathrm{Q}$ mutations are shown in orange and yellow, respectively. Protein helices, backbone and hydrogen atoms omitted for clarity. 


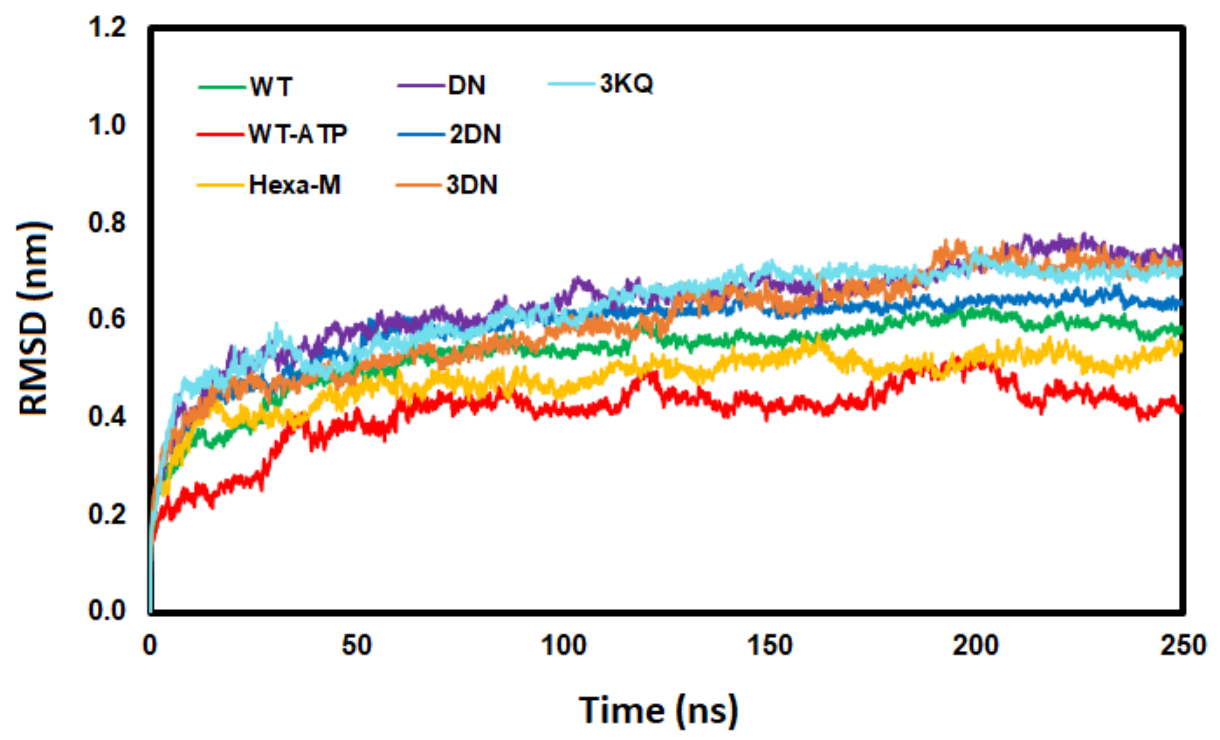

Figure S5- Root-mean-square deviation (RMSD) of the backbone atoms for the tetrameric UCP2 systems in POPC bilayer.

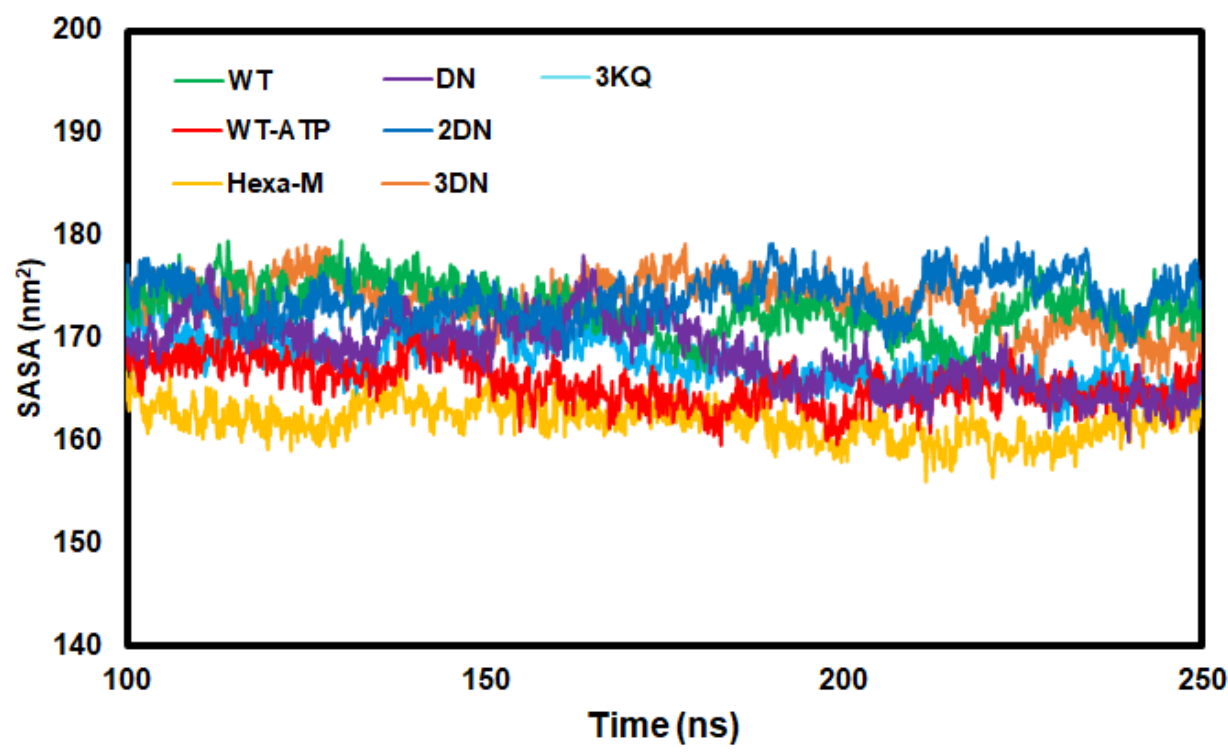

Figure S6- Normalized solvent accessible surface area (SASA) of WT and mutated UCP2 for the last $150 \mathrm{~ns}$ of simulations. As seen, in none of the systems abrupt or significant changes occur. 